DOI 10. 18307/2017. 0413

(c) 2017 by Journal of Lake Sciences

\title{
珠江三角洲养殖池塘底栖动物群落结构及水质生物学评价"
}

\author{
李志斐 ${ }^{1}$, 谢 骏 $^{1}$, 王广军 ${ }^{1}$, 林文辉 ${ }^{1}$, 余德光 ${ }^{1 * *}$, 方彰胜 ${ }^{2}$, 郁二蒙 ${ }^{1}, 弓{ }^{2}$ 凯 $^{1}$ \\ (1: 中国水产科学研究院珠江水产研究所农业部热带亚热带水产资源利用与养殖重点实验室,广州 510380) \\ (2:广东省海洋工程职业技术学校,广州 510380)
}

\begin{abstract}
摘 要: 珠江三角洲是我国主要的水产养殖区域之一. 为了解珠江三角洲鱼类养殖池塘底栖动物群落结构, 进而评价养 殖水体水质状况, 于 2016 年 7-8 月对 6 种养殖模式 30 口池塘底栖动物及其环境因子进行调查. 结果表明: 1 ) 共采集底 栖动物 18 种, 隶属于 3 门 14 属, 其中塞毛类和水生昆虫均为 7 种, 软体动物为 4 种. 优势种为克拉泊水丝蚂 ( Limnodrilus claparedeianus)、水丝蚂属一种 (Limnodrilus sp.) 和摇蚊属一种 (Chironomus sp.) 等耐污种类. 2) 6 种养殖模式池塘底栖动 物平均种类数无显著差异, 但其密度和生物量却存在显著差异, 杂交鳢 $\mathrm{Y}$ (杂交鳢幼鱼) 和杂交鳢 $\mathrm{C}$ (杂交鳢成鱼) 养殖池 塘均显著高于其他 4 种养殖模式池塘. 3) 皮尔逊相关分析结果表明, 影响珠江三角洲池塘底栖动物密度和生物量的主要 因素是总氮 $(\mathrm{TN})$ 和总磷 $(\mathrm{TP})$. 两个主要分类群中, 塞毛类密度和生物量仅与 $\mathrm{TN}$ 浓度呈显著正相关, 而摇蚊幼虫却与 $\mathrm{TN}$ 和 TP 浓度均呈显著正相关. 4) 采用 Shannon-Wiener 多样性指数、Margalef 多样性指数和 Biotic Index 生物指数对 6 种养殖 模式 30 口池塘水质进行评价, 结果表明 Shannon-Wiener 多样性指数不宜应用于珠江三角洲池塘的水质评价, Margalef 多 样性指数和 Biotic Index 生物指数的评价结果均表明杂交鳢 $\mathrm{Y}$ 、杂交鳢 $\mathrm{C}$ 以及草鱼池塘全部处于重度污染, 而大口黑鲇 $\mathrm{S}$ (投喂饲料)、大口黑鲇 $\mathrm{S}+\mathrm{B}$ (投喂饲料+冰鲜鱼类) 和罗非鱼池塘大部分处于重度污染,少数处于中度污染.
\end{abstract}

关键词: 珠江三角洲; 底栖动物; 群落结构; 水质评价

\section{Community characteristics of the macrozoobenthos and bioassessment of water quality in aquaculture ponds of the Pearl River Delta}

LI Zhifei ${ }^{1}$, XIE Jun ${ }^{1}$, WANG Guangjun ${ }^{1}$, LIN Wenhui ${ }^{1}$, YU Deguang ${ }^{1 * *}$, FANG Zhangsheng ${ }^{2}$, YU Ermeng $^{1}$ \& $^{1}$ ZHANG Kai'

(1: Key Lab of Tropical and Subtropical Fishery Resource Application and Cultivation, Ministry of Agriculture; Pearl River Fisheries Research Institute, Chinese Academy of Fishery Sciences, Guangzhou 510380, P.R.China)

(2: Vocational School of Oceanographic Engineering in Guangdong Province, Guangzhou 510380, P.R.China)

Abstract: Pearl River Delta is one of the main aquaculture areas in China. In order to understand the community structures of macrozoobenthos and further assess the water quality in aquaculture ponds, a field investigation of macrozoobenthos and environmental parameters was conducted in 30 ponds with six aquaculture models during July to August 2016 . The results showed that a total of 18 species belonging to 14 genera and 3 phyla were identified, and the species number of oligochaeta, aquatic insect, and mollusca was 7, 7, and 4, respectively. The dominant taxa of macrozoobenthos were the Limnodrilus claparedeianus, Limnodrilus sp., and Chironomus sp., which have strong tolerances to pollution. The average species number among the six model ponds had no significant differences. However, the density and biomass were significantly different, and which were significant higher in two models (juvenile and adult fish, respectively) of hybrid snakehead (Channa maculate $+\times$ Channa arguss $\$$ ) than the other four model ponds. Pearson correlation analyses showed that the TN and TP were the main factors influencing the density and biomass of macrozoobenthos in aquaculture ponds of Pearl River Delta. The density and biomass of oligochaeta were only positively correlated with TN, while which of chironomids were significantly affected by TN and TP. The water quality of 30 aquaculture ponds were assessed using Shannon-Wiener index, Margalef index, and Biotic index. It suggested that the Shannon-Wiener index was not appropriate for

* 国家科技支撑计划项目(2012BAD25B04) 和公益性行业(农业)科研专项 (201203083) 联合资助. 2016-09-24 收 稿; 2016-11-16 收修改稿. 李志斐(1983 ), 男, 助理研究员;E-mail : lzf@ prfri.ac.cn.

** 通信作者;E-mail: gzyudeguang@163.com. 
the assessment of water quality in aquaculture ponds of Pearl River Delta. The assessment results by Margalef and Biotic index showed that all the ponds of grass carp (Ctenopharyngodon idellus) and hybrid snakehead were heavily polluted, while most ponds of large mouth bass (Micropterus salmoides) and tilapia (Oreochromis mossambicus) were heavily polluted and a few ponds were moderately polluted.

Keywords: Pearl River Delta; macrozoobenthos; community structure; water quality bioassessment

珠江三角洲水系发达、池塘棋布, 是我国最大的淡水商品鱼养殖生产基地. 池塘养殖作为珠江三角洲淡 水养殖的主要类型, 更是有着悠久的历史 ${ }^{[1]}$. 然而, 近几十年来, 在高经济效益的驱动下, 区域内水产养殖无 论从养殖类型还是养殖方法上均发生了巨大变化. 从最早期的基塘养殖等粗放型模式逐渐转变为高密度、 精养型模式, 渔产量大大提高 ${ }^{[2]}$. 伴随这一转变,一系列池塘水环境问题也逐渐显现出来. 由于池塘水体的 自净和缓冲能力十分有限, 池塘中饲料的大量投放不仅使水体营养物质浓度大幅上升、还使病原微生物大 量滋生, 严重威胁鱼类健康; 而池塘中各种杀菌药物的使用, 又进一步加剧了水体的污染 ${ }^{[3]}$. 由于水环境健 康直接关系到水产品的质量安全, 因此开展珠江三角洲养殖池塘的监测与水质健康评价工作对健康养殖具 有十分重要的意义.

底栖动物是池塘生态系统的重要组成部分, 作为次级生产者, 它不但可以为鱼类提供天然饵料, 还可对 池塘底部的沉积物进行分解转化, 加速营养物质的转移, 因此在物质循环和能量流动等方面发挥着巨大作 用 ${ }^{[4]}$. 此外, 由于底栖动物包含许多敏感种和耐污种, 且具有分布广泛、生活史较长、迁移能力弱等特点, 因 此可以较好地反映外界环境的变化 ${ }^{[5]}$. 与传统的水化学监测相比, 后者只能反映水体的瞬时污染状况, 很难 全面反映水体长期的健康状况 ${ }^{[6]}$. 利用底栖动物进行生物监测不仅可以克服以上缺点, 还能反映各污染物 之间的联合或协同效应, 且具有操作简便、成本较低的特点 ${ }^{[7]}$. 因此, 底栖动物已被普遍认为是水质监测的 理想类群.

目前, 国内利用底栖动物来评价水质健康状况已开展大量工作, 但研究对象主要集中在河流 (如长 汇 $^{[8]}$ 、黄河 ${ }^{[9]} 、$ 松花江 ${ }^{[10]}$ 等)、湖泊 (如东湖 ${ }^{[11]} 、$ 洞庭湖 ${ }^{[12]}$ 等) 和水库 (如丹江口水库 ${ }^{[13]}$ ) 等大型水体. 作为 小水体的养殖池塘, 国内目前报道较少, 尚未形成共同认可的评价方法与标准 ${ }^{[2]}$. 在珠江三角洲地区, 刘乾 甫等 ${ }^{[2]}$ 利用水化学方法初步评价了 4 种养殖池塘的水质状况, 但关于水生生物的评价至今未见报道. 考虑 到高密度养殖池塘夏季经常发生一些突发性事件, 导致鱼类在短时间内大量死亡. 因此, 本研究选择夏季 (7-8 月) 对珠江三角洲 4 种养殖鱼类、6 种养殖模式池塘底栖动物进行调查, 分析池塘底栖动物的群落结 构以及主要影响因素, 并对池塘水质进行生物学评价. 本研究结果不仅对池塘水质的净化与调控具有重要 意义,还可为池塘的生态管理提供理论依据.

\section{1 研究区域与方法}

\section{1 研究区域概况}

珠江三角洲位于珠江下游、广东省东南部,包括广州、深圳、佛山、东莞、中山、珠海、江门、肇庆、惠州等 9 个城市, 与东南亚隔海相望, 被称为中国的 “南大门”. 珠江三角洲是西江、北江共同冲积形成的大三角洲与 东江冲积形成的小三角洲的总称, 面积约 $11000 \mathrm{~km}^{2}$. 区域内地势起伏较大, 四周是丘陵、山地和岛屿, 中部 是平原. 气候以南亚热带季风气候为主, 高温多雨.

珠江三角洲水网密布、池塘集中连片, 水产养殖十分发达. 根据其年产量的大小, 主要养殖对象有草鱼 (Ctenopharyngodon idellus)、罗非鱼 (Oreochromis mossambicus)、大口黑鲈 (Micropterus salmoides)、杂交鳢 (Channa maculate $9 \times$ Channa arguss $\hat{\delta}$ )、笋壳鱼 (Oxyeleotris marmoratus) 和鳢 (Siniperca chuatsi) 等. 本研究选 择草鱼、罗非鱼、大口黑鲇和杂交鳢 4 种鱼类养殖池塘作为研究对象, 对 30 口池塘底栖动物进行了采集. 其 中草鱼共选择 5 口池塘, 罗非鱼共选择 6 口池塘, 大口黑鲇共选择 9 口池塘(其中投喂饲料池塘 5 口, 投喂饲 料+冰鲜鱼类池塘 4 口), 杂交鳢池塘共选择 10 口(其中杂交鳢幼鱼池塘 5 口, 杂交鳢成鱼池塘 5 口). 每口 池塘采用五点采样法, 即池塘四角和中心 5 个点采样, 然后混合为一个样. 根据养殖对象的规格、投喂饵料 类型等, 4 种鱼类池塘可分为 6 种养殖模式,其基本概况见表 1 ,采样区域分布见图 1 . 


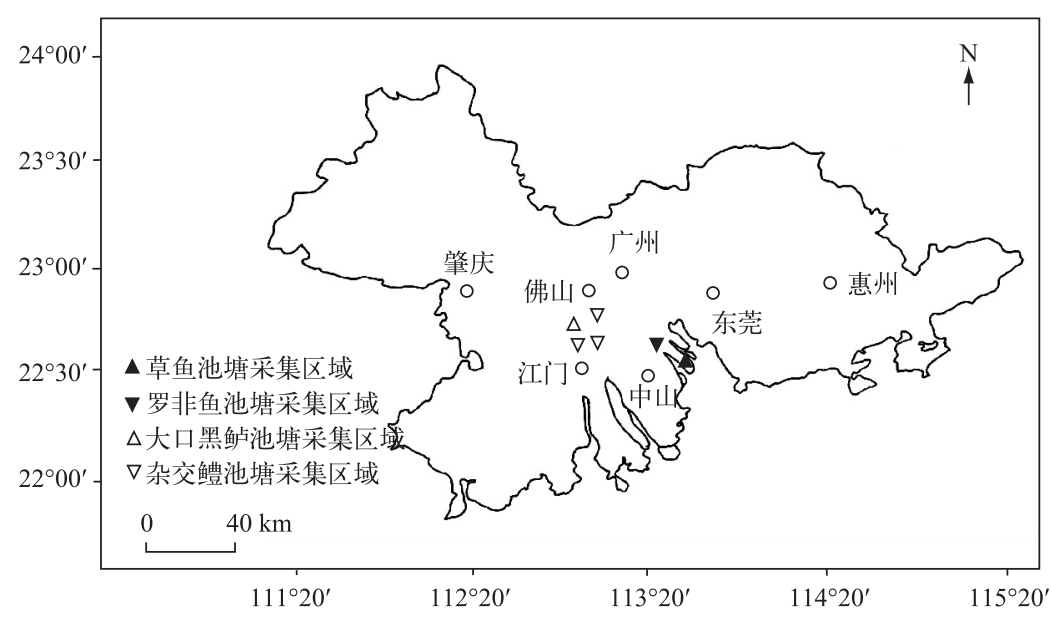

图 1 珠江三角洲不同类型养殖池塘采样区域分布

Fig. 1 Distribution of sampling areas of different aquaculture model ponds in Pearl River Delta

表 1 珠江三角洲 6 种养殖模式池塘概况

Tab.1 Overview of the six aquaculture model ponds in Pearl River Delta

\begin{tabular}{lccccccc}
\hline 养殖模式 & \multirow{2}{*}{ 养殖规格 } & 食物类型 & 营养成分 & $\begin{array}{c}\text { 池塘面积 } \\
\mathrm{hm}^{2}\end{array}$ & $\begin{array}{c}\text { 渔产量/ } \\
\left(\mathrm{kg} /\left(\mathrm{hm}^{2} \cdot \mathrm{a}\right)\right)\end{array}$ & $\begin{array}{c}\text { 调查池塘 } \\
\text { 数量 }\end{array}$ & $\begin{array}{c}\text { 平均 } \\
\text { 水深 } / \mathrm{m}\end{array}$ \\
\hline 草鱼 & $40 \sim 45 \mathrm{~cm}$ & 饲料 & 粗蛋白 $\geqslant 26 \% ; \mathrm{TP} \geqslant 0.6 \%$ & 0.667 & $3.75 \times 10^{4}$ & 5 & 3.0 \\
罗非鱼 & $10 \sim 15 \mathrm{~cm}$ & 饲料 & 粗蛋白 $\geqslant 30 \% ; \mathrm{TP} \geqslant 0.7 \%$ & 0.400 & $2.10 \times 10^{4}$ & 6 & 2.1 \\
大口黑鲇 $\mathrm{S}$ & $12 \sim 15 \mathrm{~cm}$ & 饲料 & 粗蛋白 $\geqslant 42 \% ; \mathrm{TP} \geqslant 1.0 \%$ & 0.467 & $6.00 \times 10^{4}$ & 5 & 2.9 \\
大口黑鲇 $\mathrm{S}+\mathrm{B}$ & $12 \sim 15 \mathrm{~cm}$ & 饲料+冰鲜鱼类 & 粗蛋白 $\geqslant 42 \% ; \mathrm{TP} \geqslant 1.0 \%$ & 0.467 & $6.00 \times 10^{4}$ & 4 & 2.6 \\
杂交鳢 $\mathrm{Y}$ & $6 \sim 7 \mathrm{~cm}$ & 饲料 & 粗蛋白 $\geqslant 40 \% ; \mathrm{TP} \geqslant 1.0 \%$ & 0.333 & $9.75 \times 10^{4}$ & 5 & 1.9 \\
杂交鳢 $\mathrm{C}$ & $35 \sim 40 \mathrm{~cm}$ & 饲料 & 粗蛋白 $\geqslant 40 \% ; \mathrm{TP} \geqslant 1.0 \%$ & 0.333 & $9.75 \times 10^{4}$ & 5 & 1.9 \\
\hline
\end{tabular}

\section{2 研究方法}

于 2016 年 7-8 月对珠江三角洲地区 6 种养殖模式池塘底栖动物和相关环境因子进行调查. 在每口池 塘的中部, 首先用测深锤测定池塘的水深, 用 YSI 多功能水质分析仪 (Pro Plus) 测定水体的水温 ( Temp)、溶 解氧( DO) 、电导率( Cond) 、 $\mathrm{pH}$ 、总溶解性固体物质( TDS) 等指标, 然后采用 $1 / 16 \mathrm{~m}^{2}$ 的彼得森采泥器对底栖 动物进行采集. 样品经 60 目篮网篮洗干净后, 置于白色解剖盘中逐一挑出底栖动物标本, 并用 $10 \%$ 的福尔 马林溶液加以固定. 标本带回实验室后进行鉴定、计数和称重. 根据相关资料 ${ }^{[14-16]}$, 大部分物种鉴定到属或 种, 少部分鉴定到目或科. 底栖动物湿重的测定采用万分之一天平, 先用滤纸吸去标本的表面液体, 然后置 于电子天平上称重. 最后根据采样面积计算每口池塘底栖动物的密度和生物量, 并以相对密度 $\geqslant 5 \%$ 作为优 势种的标准 ${ }^{[17]}$. 为了解池塘水体的营养状况, 每口池塘均取混合水样, 带回实验室按照标准方法 ${ }^{\left[{ }^{18]}\right.}$ 测定水 体中的总氮 $(\mathrm{TN}) 、$ 总磷 $(\mathrm{TP})$ 、磷酸盐 $\left(\mathrm{PO}_{4}^{3-}-\mathrm{P}\right)$ 和总有机碳 $(\mathrm{TOC})$ 浓度等指标.

\section{3 数据分析}

运用单因素方差分析 (One-way ANOVA) 来检验 6 种养殖模式池塘环境因子与底栖动物密度、生物量之 间差异的显著性. 采用 Duncan's 多重比较检验组间差异. 采用皮尔逊相关分析来检验底栖动物种类数、不同 类群密度和生物量与主要环境因子的相关性, 如果 $P<0.05$, 则认为有显著相关关系. 以上所有分析在 SPSS 13.0 软件中完成.

采用 Shannon-Wiener 多样性指数 $\left(H^{\prime}\right)$ 、Margalef 多样性指数 $(D)$ 和 Biotic $\operatorname{Index}(B I)$ 生物指数来评价养 殖池塘水体的健康状况, 其计算公式及评价标准如下:

1) $H^{\prime}=-\Sigma\left(P_{i}\right)\left(\log _{2} P_{i}\right)$, 式中 $P_{i}$ 为物种 $i$ 个体数所占的比例. 其评价标准为: $0 \sim 1$ (重度污染)、1 2(中 
度污染)、2 3(轻度污染) 、 $>3(\text { 清洁 })^{[19]}$.

2) $D=(S-1) / \ln N$, 式中 $N$ 为样品中所有物种的总个体数, $S$ 为样品中种类总数. 其评价标准为: $0 \sim 1$ (重度污染)、1 2(中度污染) 、2 3( 轻度污染) 、 3 (清洁 ${ }^{[20]}$.

3) $B I=\sum N_{i} T_{i} / N$, 式中 $N_{i}$ 为第 $i$ 个分类单元的个体数, $T_{i}$ 为第 $i$ 个分类单元的耐污值 ${ }^{[7,21]}, N$ 为样本总个 体数. 其评价标准为: $>8.80$ (重度污染)、7.71 8.80 (中度污染)、6.61 7.70(轻度污染)、 $5.50 \sim 6.60$ (清洁)、 $<5.50$ (最清洁 $)^{[7]}$.

\section{2 研究结果}

\section{1 环境特征}

珠江三角洲 6 种养殖模式池塘环境参数中, 除了 $\mathrm{TOC}$ 和 $\mathrm{PO}_{4}^{3-}-\mathrm{P}$ 浓度没有显著差异外, 其余参数均存在 显著差异 (表 2). 与其他类型池塘相比草鱼池塘的 DO 浓度最低, 但其 Cond 和 TDS 浓度却显著高于其他池 塘. 罗非鱼池塘的 $\mathrm{pH}$ 与大口黑鲇 $\mathrm{S}$ 池塘差异不大, 却显著高于其他 4 种养殖模式池塘. 就营养盐浓度而言, 杂交鳢 $\mathrm{Y}$ 和杂交鳢 $\mathrm{C}$ 池塘 TN 和 TP 浓度较高, 且 TN 浓度均显著高于其他池塘. 杂交鳢 $\mathrm{Y}$ 池塘的 TP 浓度显 著高于其他 4 种养殖模式池塘, 但杂交鳢 $\mathrm{C}$ 池塘 TP 浓度却与大口黑鲇 $\mathrm{S}$ 池塘并无显著差异.

以《地表水环境质量标准》(GB 3838-2002) 作为评价标准, 6 种养殖模式池塘 TN 和 TP 浓度平均值均 远超 $\mathrm{V}$ 类水质标准. 对 30 口池塘而言,仅有 1 个罗非鱼池塘属于 $\mathrm{IV}$ 类水,其余均为 $\mathrm{V}$ 类水.

\section{表 2 珠江三角洲 6 种养殖模式池塘环境参数}

Tab.2 Environmental parameters of six aquaculture model ponds in Pearl River Delta

\begin{tabular}{lccccccc}
\hline 环境参数 & 草鱼 & 罗非鱼 & 大口黑鲈 $\mathrm{S}$ & 大口黑鲈 $\mathrm{S}+\mathrm{B}$ & 杂交鳢 $\mathrm{Y}$ & 杂交鳢 $\mathrm{C}$ & $F$ 值 \\
\hline $\mathrm{Temp} /{ }^{\circ} \mathrm{C}$ & $32.14 \pm 0.11^{\mathrm{a}}$ & $33.68 \pm 0.83^{\mathrm{c}}$ & $32.66 \pm 0.57^{\mathrm{ab}}$ & $32.6 \pm 0.70^{\mathrm{ab}}$ & $33.94 \pm 0.80^{\mathrm{c}}$ & $33.34 \pm 0.55^{\mathrm{bc}}$ & $5.92^{* *}$ \\
$\mathrm{DO} /(\mathrm{mg} / \mathrm{L})$ & $4.19 \pm 0.31^{\mathrm{a}}$ & $7.29 \pm 2.78^{\mathrm{b}}$ & $6.49 \pm 2.56^{\mathrm{b}}$ & $4.52 \pm 0.35^{\mathrm{a}}$ & $6.23 \pm 3.42^{\mathrm{b}}$ & $6.21 \pm 2.47^{\mathrm{b}}$ & $3.59^{*}$ \\
$\mathrm{Cond} /(\mu \mathrm{S} / \mathrm{cm})$ & $762.20 \pm 162.12^{\mathrm{a}}$ & $270.53 \pm 108.39^{\mathrm{b}}$ & $370.9 \pm 52.33^{\mathrm{b}}$ & $435.5 \pm 53.94^{\mathrm{b}}$ & $328.14 \pm 81.69^{\mathrm{b}}$ & $409.48 \pm 177.37^{\mathrm{b}} 11.07^{* * *}$ \\
$\mathrm{TDS} /(\mathrm{mg} / \mathrm{L})$ & $495.20 \pm 105.75^{\mathrm{a}}$ & $175.72 \pm 70.58^{\mathrm{b}}$ & $241 \pm 33.86^{\mathrm{b}}$ & $281.75 \pm 34.94^{\mathrm{b}}$ & $206.81 \pm 52.89^{\mathrm{b}}$ & $266.54 \pm 116.03^{\mathrm{b}} 11.12^{* * * *}$ \\
$\mathrm{pH}$ & $7.64 \pm 0.12^{\mathrm{ab}}$ & $8.32 \pm 0.74^{\mathrm{c}}$ & $8.04 \pm 0.35^{\mathrm{bc}}$ & $7.70 \pm 0.22^{\mathrm{ab}}$ & $7.63 \pm 0.43^{\mathrm{ab}}$ & $7.35 \pm 0.14^{\mathrm{a}}$ & $3.70^{*}$ \\
$\mathrm{TOC} /(\mathrm{mg} / \mathrm{L})$ & $15.04 \pm 2.37$ & $13.35 \pm 3.15$ & $15.14 \pm 2.25$ & $13.4 \pm 0.79$ & $17.14 \pm 5.05$ & $16.96 \pm 3.85$ & 1.28 \\
$\mathrm{TN} /(\mathrm{mg} / \mathrm{L})$ & $4.43 \pm 1.74^{\mathrm{a}}$ & $5.13 \pm 3.86^{\mathrm{a}}$ & $3.99 \pm 1.12^{\mathrm{a}}$ & $5.28 \pm 5.24^{\mathrm{a}}$ & $9.71 \pm 0.97^{\mathrm{b}}$ & $10.56 \pm 3.56^{\mathrm{b}}$ & $4.29^{* *}$ \\
$\mathrm{TP} /(\mathrm{mg} / \mathrm{L})$ & $0.38 \pm 0.06^{\mathrm{a}}$ & $0.39 \pm 0.19^{\mathrm{a}}$ & $0.51 \pm 0.29^{\mathrm{ab}}$ & $0.35 \pm 0.13^{\mathrm{a}}$ & $1.20 \pm 0.43^{\mathrm{c}}$ & $0.85 \pm 0.54^{\mathrm{bc}}$ & $5.68^{* *}$ \\
$\mathrm{PO} 4^{3-}-\mathrm{P} /(\mathrm{mg} / \mathrm{L})$ & $0.22 \pm 0.11$ & $0.20 \pm 0.22$ & $0.38 \pm 0.29$ & $0.27 \pm 0.19$ & $0.22 \pm 0.12$ & $0.19 \pm 0.04$ & 0.73 \\
\hline
\end{tabular}

表中数值为平均值士标准差. 上标不同小写字母表示存在显著差异; $* P<0.05, * * P<0.01, * * * P<0.001$.

\section{2 种类组成}

共采集底栖动物 18 种, 隶属于 3 门 14 属, 其中寡毛类和水生昆虫均为 7 种, 软体动物为 4 种 (表 3 ). 6 种养殖模式池塘中, 罗非鱼池塘种类数最多, 为 11 种; 其次是 2 种大口黑鲇池塘, 均为 10 种; 草鱼和杂交鳢 $\mathrm{C}$ 池塘种类数最少, 均为 8 种 (表 3 ). 方差分析结果表明, 6 种养殖模式池塘平均种类数无显著差异 $(F=$ $1.27, P=0.31)$.

30 口池塘底栖动物优势种为克拉泊水丝蚓、水丝蚓属的一种和摇蚊属的一种等耐污种类. 6 种养殖模 式池塘底栖动物优势种具有一定差异, 其种类组成见表 4. 草鱼池塘优势种最单一, 仅有克拉伯水丝蚂和水 丝蚓 2 种; 大口黑鲇 $\mathrm{S}+\mathrm{B}$ 池塘优势种最多样, 分别为水丝蚓、苏氏尾鰓蚓、霍甫水丝蚓、摇蚊和长足摇蚊 5 种; 其余 4 种养殖模式池塘优势种均为 3 种.

\section{3 密度和生物量}

珠江三角洲池塘底栖动物平均密度为 $14331 \mathrm{ind} . / \mathrm{m}^{2}$, 变化范围为 $96 \sim 97280 \mathrm{ind} . / \mathrm{m}^{2}$; 平均生物量为 $35.53 \mathrm{~g} / \mathrm{m}^{2}$, 变化范围为 $0.09 \sim 214.53 \mathrm{~g} / \mathrm{m}^{2}$. 不同分类群中, 寡毛类和水生昆虫密度分别为 6191 和 8131 ind. $/ \mathrm{m}^{2}$, 生物量分别为 16.97 和 $16.94 \mathrm{~g} / \mathrm{m}^{2}$, 二者之和占总密度或总生物量的 $95 \%$ 以上. 3 个优势种密度和 生物量之和分别为 12693 ind. $/ \mathrm{m}^{2}$ 和 $23.33 \mathrm{~g} / \mathrm{m}^{2}$, 分别占总密度或总生物量的 $88.6 \%$ 和 $68.8 \%$ (表 4). 
表 3 珠江三角洲 6 种养殖模式池塘底栖动物种类组成

Tab.3 Species composition of macrozoobenthos in six aquaculture model ponds of Pearl River Delta

\begin{tabular}{|c|c|c|c|c|c|c|}
\hline 分类单元 & 草鱼 & 罗非鱼 & 大口黑鲈 S & 大口黑鲈 S+B & 杂交鳢 Y & 杂交鳢 C \\
\hline \multicolumn{7}{|l|}{ 塞毛纲 Oligochaeta } \\
\hline 仙女虫属一种 Nais sp. & & & & & + & \\
\hline 苏氏尾鳃蚓 Branchiura sowerbyi & + & + & + & + & + & + \\
\hline 霍甫水丝蚓 Limnodrilus hoffmeisteri & + & + & & + & & \\
\hline 克拉泊水丝蚓 Limnodrilus claparedeianus & + & + & + & + & + & + \\
\hline 巨毛水丝蚓 Limnodrilus grandisetosus & & + & & + & + & \\
\hline 水丝蚓属一种 Limnodrilus sp. & + & + & + & + & + & + \\
\hline 皮氏管水蚓 Aulodrilus pigueti & & & + & & & \\
\hline \multicolumn{7}{|l|}{ 水生昆虫 Aquatic Insecta } \\
\hline 四节蜉属一种 Baetis sp. & & & + & & & \\
\hline 双翅目一种 Diptera sp. & & + & & + & + & + \\
\hline 摇蚊属一种 Chironomus sp. & + & + & + & + & + & + \\
\hline 小摇蚊属一种 Microchironomus sp. & + & & + & + & + & + \\
\hline 长足摇蚊属一种 Tanypus sp. & + & + & + & + & + & + \\
\hline 长足摇蚊 Tanypus concavus & + & & & & & \\
\hline 异腹鳃摇蚊属一种 Einfeldia $\mathrm{sp.}$ & & + & + & & & \\
\hline \multicolumn{7}{|l|}{ 软体动物 Mollusca } \\
\hline 长角涵螺 Alocinma longicornis & & + & & & & \\
\hline 放逸短沟蜷 Semisulcospira libertina & & + & & + & & \\
\hline 纹沼螺 Parafossarulus striatulus & & & + & & & \\
\hline 膀胱螺科一种 Physidae & & & & & & + \\
\hline 合计 & 8 & 11 & 10 & 10 & 9 & 8 \\
\hline
\end{tabular}

6 种养殖模式池塘底栖动物密度和生物量均存在显著差异; 杂交鳢 $\mathrm{Y}$ 和杂交鳢 $\mathrm{C}$ 养殖池塘之间差异不 显著, 但却均显著高于其他 4 种养殖模式池塘 (图 2). 6 种养殖池塘不同底栖动物类群密度和生物量所占的 比例也有较大差异. 草鱼、罗非鱼和杂交鳢 $\mathrm{C}$ 池塘中, 寡毛类密度所占比例要明显高于水生昆虫和软体动 物, 而其余 3 种模式池塘中水生昆虫密度所占比例却是最高的 (图 3). 生物量所占比例方面, 草鱼、大口黑 鲇 $\mathrm{S}$ 、大口黑鲇 $\mathrm{S}+\mathrm{B}$ 池塘中寡毛类最高, 杂交鳢 $\mathrm{Y}$ 和杂交鳢 $\mathrm{C}$ 池塘中水生昆虫最高, 而罗非鱼池塘中软体动 物最高 (图 4).
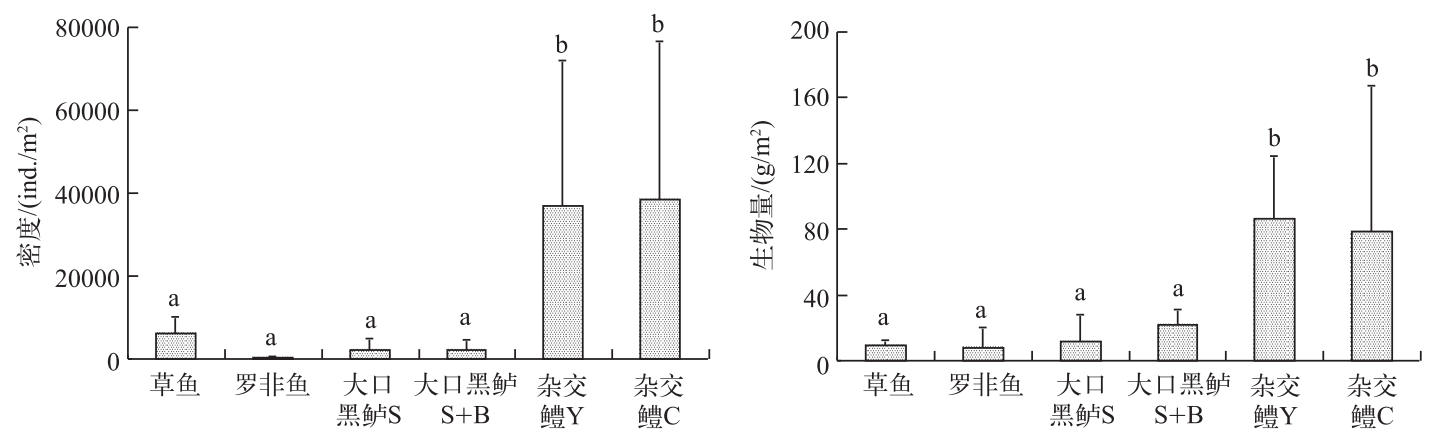

图 2 珠江三角洲 6 种养殖模式池塘底栖动物密度和生物量对比

Fig.2 Comparison of the density and biomass of macrozoobenthos in six aquaculture model ponds of Pearl River Delta 
表 4 珠江三角洲 6 种底栖动物优势种相对丰度、密度和生物量

Tab.4 Relative abundance, density, and biomass of the dominant species in six aquaculture model ponds of Pearl River Delta

\begin{tabular}{|c|c|c|c|c|c|c|c|}
\hline 养殖模式池塘 & & 克拉泊水丝蚓 & 水丝蚓属一种 & 苏氏尾鳃蚓 & 霍甫水丝蚓 & 摇蚊属一种 & 长足摇蚊属一种 \\
\hline \multirow[t]{3}{*}{ 全部池塘 } & 相对丰度 & $6.87 \%$ & $30.43 \%$ & & & $51.28 \%$ & \\
\hline & 密度 $/\left(\right.$ ind. $\left./ \mathrm{m}^{2}\right)$ & 985 & 4360 & & & 7348 & \\
\hline & 生物量 $/\left(\mathrm{g} / \mathrm{m}^{2}\right)$ & 1.87 & 6.17 & & & 15.29 & \\
\hline \multirow[t]{3}{*}{ 草鱼 } & 相对丰度 & $10.15 \%$ & $84.72 \%$ & & & & \\
\hline & 密度/( ind. $\left./ \mathrm{m}^{2}\right)$ & 634 & 5286 & & & & \\
\hline & 生物量 $/\left(\mathrm{g} / \mathrm{m}^{2}\right)$ & 1.07 & 7.62 & & & & \\
\hline \multirow[t]{3}{*}{ 罗非鱼 } & 相对丰度 & $9.29 \%$ & $30.00 \%$ & $46.43 \%$ & & & \\
\hline & 密度 $/\left(\right.$ ind.$\left./ \mathrm{m}^{2}\right)$ & 35 & 112 & 173 & & & \\
\hline & 生物量 $/\left(\mathrm{g} / \mathrm{m}^{2}\right)$ & 0.03 & 0.12 & 2.18 & & & \\
\hline \multirow[t]{3}{*}{ 大口黑鲈 S } & 相对丰度 & & $19.97 \%$ & $11.19 \%$ & & & $56.16 \%$ \\
\hline & 密度 $/\left(\right.$ ind.$\left./ \mathrm{m}^{2}\right)$ & & 418 & 234 & & & 1177 \\
\hline & 生物量/ $\left(\mathrm{g} / \mathrm{m}^{2}\right)$ & & 0.28 & 2.13 & & & 0.86 \\
\hline \multirow[t]{3}{*}{ 大口黑鲈 S+B } & 相对丰度 & & $15.19 \%$ & $14.31 \%$ & $10.60 \%$ & $7.77 \%$ & $37.63 \%$ \\
\hline & 密度 $/\left(\right.$ ind.$\left./ \mathrm{m}^{2}\right)$ & & 344 & 324 & 240 & 176 & 852 \\
\hline & 生物量 $/\left(\mathrm{g} / \mathrm{m}^{2}\right)$ & & 0.36 & 8.46 & 0.29 & 2.06 & 3.24 \\
\hline \multirow[t]{3}{*}{ 杂交鳢 Y } & 相对丰度 & & $12.16 \%$ & $6.85 \%$ & & $72.80 \%$ & \\
\hline & 密度/( ind. $\left./ \mathrm{m}^{2}\right)$ & & 4506 & 2538 & & 26972 & \\
\hline & 生物量 $/\left(\mathrm{g} / \mathrm{m}^{2}\right)$ & & 6.44 & 30.30 & & 45.86 & \\
\hline \multirow[t]{3}{*}{ 杂交鳢 C } & 相对丰度 & $10.81 \%$ & $40.54 \%$ & & & $43.98 \%$ & \\
\hline & 密度 $/\left(\right.$ ind.$\left./ \mathrm{m}^{2}\right)$ & 4144 & 15542 & & & 16861 & \\
\hline & 生物量 $/\left(\mathrm{g} / \mathrm{m}^{2}\right)$ & 8.72 & 21.57 & & & 43.73 & \\
\hline
\end{tabular}

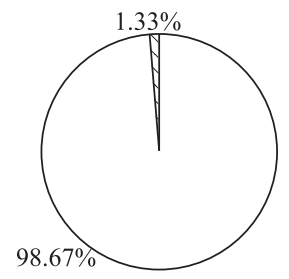

草鱼

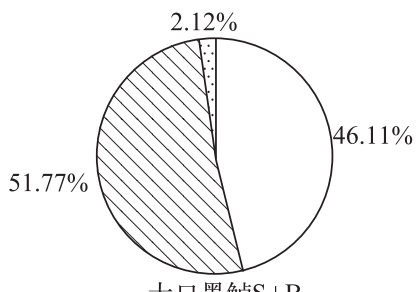

大口黑鲇 $\mathrm{S}+\mathrm{B}$

口寡毛类 $\square$ 水生昆虫 $\square$ 软体动物

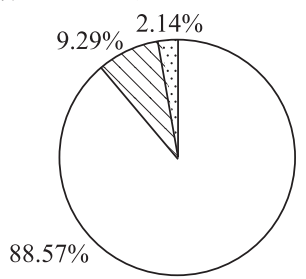

罗非鱼

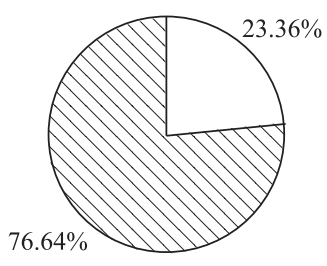

杂交鳢 $Y$

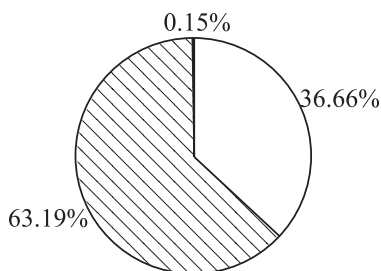

大口黑鲇S



杂交鳢 $\mathrm{C}$

图 3 珠江三角洲 6 种养殖模式池塘底栖动物分类群密度所占比例

Fig.3 Density percentage of each taxonomic group of macrozoobenthos in six aquaculture model ponds of Pearl River Delta 

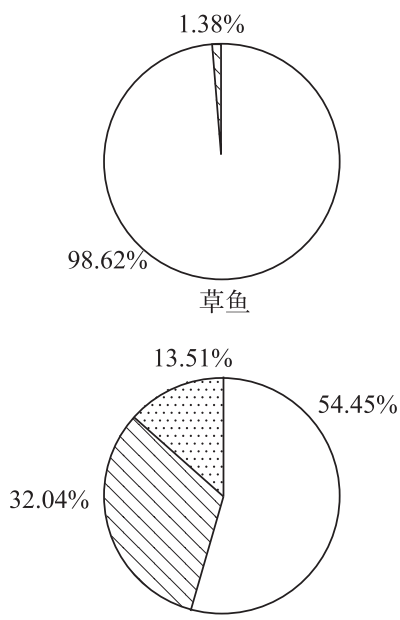

大口黑鲇 $\mathrm{S}+\mathrm{B}$

口寡毛类 曰水生昆虫 $\square$ 软体动物
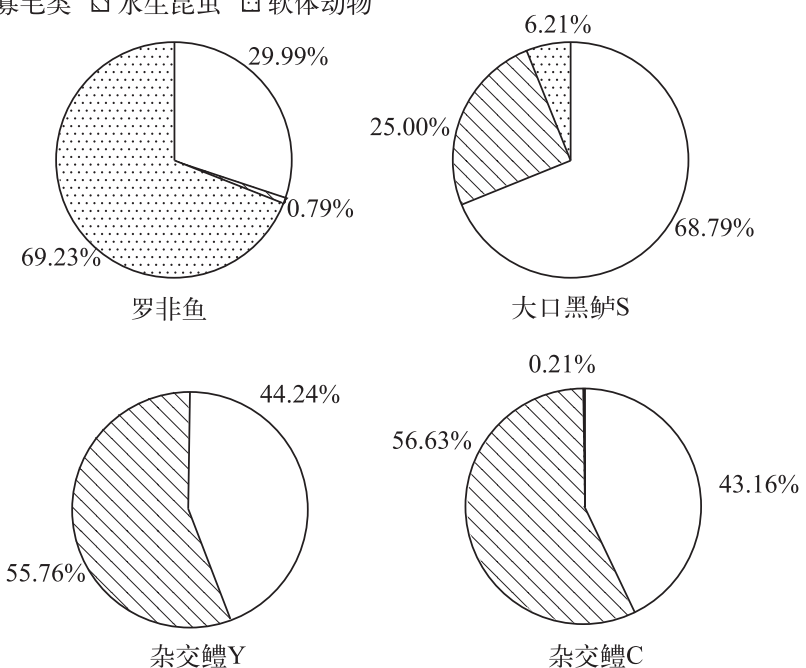

图 4 珠江三角洲 6 种养殖模式池塘底栖动物分类群生物量所占比例

Fig.4 Biomass percentage of each taxonomic group of macrozoobenthos in six aquaculture model ponds of Pearl River Delta

\section{4 影响因素}

表 4 显示了珠江三角洲底栖动物种类数、密度、生物量以及主要分类群寡毛类和摇蚊幼虫密度、生物量 与环境因子的皮尔逊相关关系. 结果表明底栖动物种类数与环境因子并无相关关系, 而密度和生物量却均 与 TN 和 TP 浓度呈显著正相关. 两个分类群中, 寡毛类密度和生物量仅与 TN 呈显著正相关, 而摇蚊幼虫密 度却与 TN 和 TP 均呈显著正相关(表 5).

表 5 底栖动物种类数、密度和生物量与环境因子的皮尔逊相关关系

Tab.5 Pearson correlation between environmental parameters and species number, density and biomass of the macrozoobenthos

\begin{tabular}{|c|c|c|c|c|c|c|c|c|c|c|c|c|c|c|c|c|}
\hline & 种类数 & 密度 & 生物量 & Den $_{\text {Oli }}$ & $\mathrm{Bio}_{\mathrm{Oli}} \mathrm{I}$ & Den $_{\text {Chiron }}$ & ${ }_{n} \mathrm{Bio}_{\text {Chiron }}$ & n Temp & DO & Cond & TDS & $\mathrm{pH}$ & $\mathrm{PO}_{4}^{3--}-\mathrm{P}$ & TOC & $\mathrm{TN}$ & $\mathrm{TP}$ \\
\hline 种类数 & 1.00 & & & & & & & & & & & & & & & \\
\hline 密度 & 0.26 & 1.00 & & & & & & & & & & & & & & \\
\hline 生物量 & 0.36 & $0.83^{* * *}$ & $* * 1.00$ & & & & & & & & & & & & & \\
\hline Den $_{\text {Oli }}$ & 0.26 & $0.57^{\text {** }}$ & 0.62 & $* * 1.00$ & & & & & & & & & & & & \\
\hline $\mathrm{Bio}_{\mathrm{Oli}}$ & $0.49^{\text {** }}$ & 0.42 & $0.67^{* * *}$ & *** 0.77 *** & *** 1.00 & & & & & & & & & & & \\
\hline Den $_{\text {Chiron }}$ & 0.18 & $0.91^{* * * *}$ & 0.68 & $* * 0.18$ & 0.12 & 1.00 & & & & & & & & & & \\
\hline $\mathrm{Bio}_{\text {Chiron }}$ & 0.23 & $0.84^{* * * *}$ & 0.89 & $* * 0.34$ & 0.32 & $0.84^{* * *}$ & $* * 1.00$ & & & & & & & & & \\
\hline Temp & 0.23 & 0.20 & 0.31 & 0.26 & $0.42 *$ & 0.10 & 0.15 & 1.00 & & & & & & & & \\
\hline DO & 0.19 & 0.12 & 0.10 & 0.08 & 0.20 & 0.10 & 0.01 & $0.64^{* * * *}$ & 1.00 & & & & & & & \\
\hline Cond & -0.13 & 0.01 & -0.13 & 0.19 & -0.09 & -0.07 & -0.11 & $-0.59 * *$ & -0.65 * & ***** 1.00 & & & & & & \\
\hline TDS & -0.13 & 0.01 & -0.13 & 0.19 & -0.08 & -0.08 & -0.12 & -0.59 ** & -0.63 & ****** 1.00 *** & ***** 1.00 & & & & & \\
\hline $\mathrm{pH}$ & -0.03 & -0.30 & -0.26 & -0.35 & -0.26 & -0.19 & -0.24 & 0.15 & 0.59 * & $* *-0.31$ & -0.30 & 1.00 & & & & \\
\hline $\mathrm{PO}_{4}^{3--} \mathrm{P}$ & 0.19 & -0.20 & -0.17 & -0.14 & 0.11 & -0.17 & -0.16 & -0.09 & 0.13 & -0.17 & -0.17 & 0.19 & 1.00 & & & \\
\hline TOC & 0.21 & 0.30 & 0.15 & $0.39^{*}$ & 0.21 & 0.17 & 0.12 & 0.23 & 0.08 & 0.22 & 0.22 & -0.24 & 0.02 & 1.00 & & \\
\hline $\mathrm{TN}$ & 0.16 & 0.53 ** & 0.60 ** & 0.46 & 0.37 * & $0.40 *$ & $0.56^{* *}$ & 0.26 & 0.02 & -0.11 & -0.11 & -0.32 & -0.01 & $0.50 * *$ & 1.00 & \\
\hline $\mathrm{TP}$ & 0.23 & $0.69^{* * *}$ & $0.66 * * *$ & $* * 0.19$ & 0.24 & $0.73^{* * *}$ & *** 0.71 ***** & *** 0.16 & -0.05 & -0.18 & -0.20 & -0.39 * & $*-0.07$ & $0.36^{*}$ & 0.59 ** & 1.00 \\
\hline
\end{tabular}

$\mathrm{Den}_{\mathrm{Oli}}$ : 寡毛类密度; $\mathrm{Bio}_{\mathrm{Oli}}$ : 塞毛类生物量, $\mathrm{Den}_{\mathrm{Chiron}}$ : 摇蚊幼虫密度, $\mathrm{Bio}_{\mathrm{Chiron}}$ : 摇蚊幼虫生物量; $*$ 表示 $P<0.05 ; * *$ 表 示 $P<0.01$; $* * *$ 表示 $P<0.001$. 


\section{5 水质评价}

Shannon-Wiener 多样性指数评价结果表明有 3 口池塘处于轻度污染, 其余均处于中度或重度污染; Margalef 多样性指数评价结果表明, 有 7 口池塘处于中度污染, 其余均处于重度污染; $B I$ 生物指数评价结果表 明有 5 口池塘处于中度污染, 其余均处于重度污染 (图 5). 相比较而言, Margalef 多样性指数和 $B I$ 生物指数 评价方法要优于 Shannon-Wiener 多样性指数.
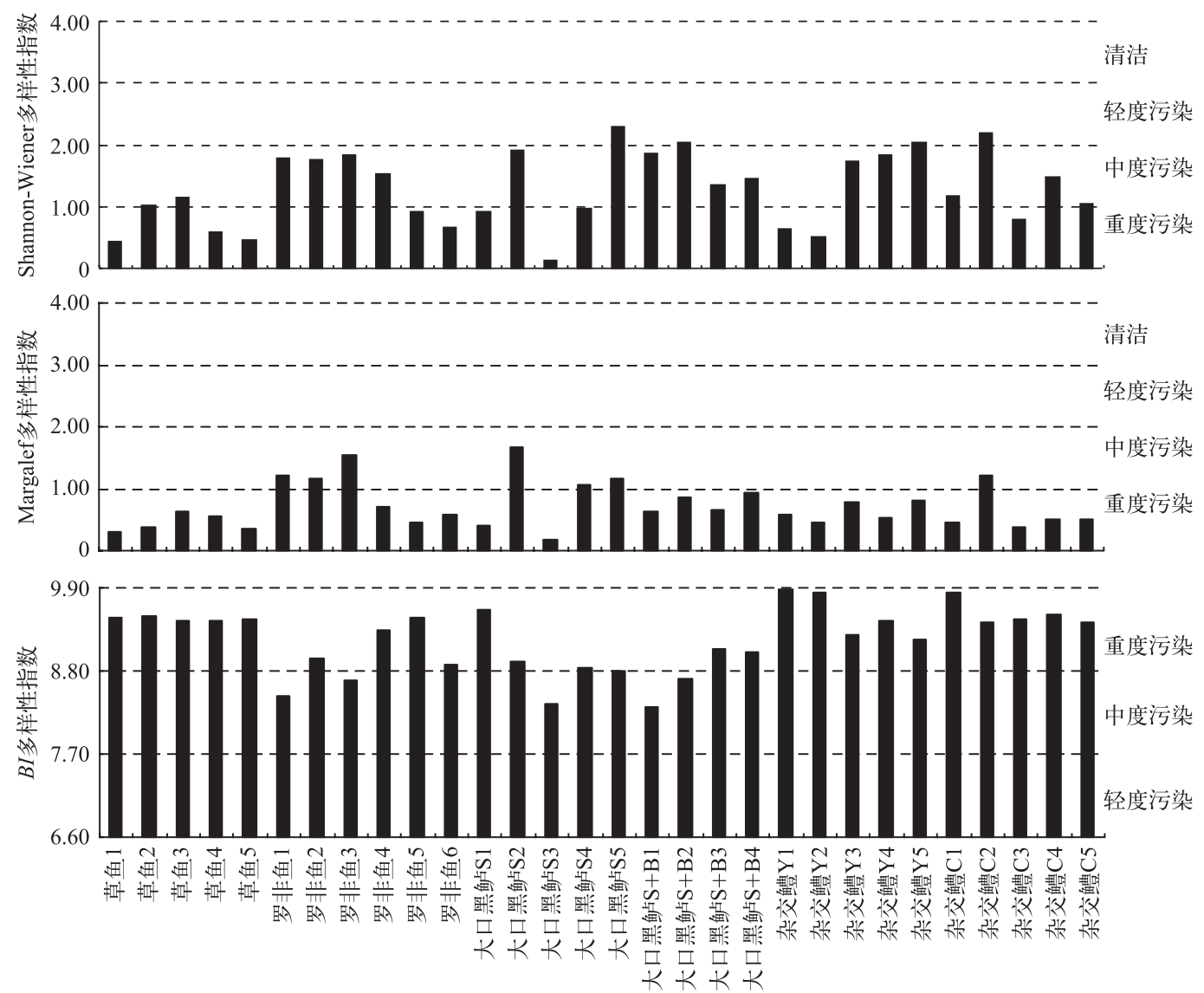

图 5 不同评价指数评价结果对比

Fig.5 Comparison of the assessment results using different assessment methods

\section{3 讨论}

\section{1 底栖动物群落结构}

珠江三角洲多为高密度养殖池塘. 与河流、湖泊等淡水水体相比, 池塘的主要功能是养殖, 这也就决定 了池塘本身要承受更高的营养负荷. 国内外一些研究已经表明, 池塘中残饵、鱼类排泄物等是池塘 N、P 以及 有机污染物的主要来源 ${ }^{[3,22]}$, 因此高密度养殖池塘的大量投饵必定会造成水体的严重富营养化. 本次调查 中 30 口池塘 TN 和 TP 浓度平均值分别高达 6.51 和 $0.61 \mathrm{mg} / \mathrm{L}$, 就很好地证明了这一点. 与之相对应, 珠江 三角洲养殖池塘底栖动物种类组成表现出种类单一、且多为耐污种的特点. 30 口池塘共采集底栖动物 18 种, 且主要是寡毛类和摇蚊幼虫. 与长江中下游一些严重富营养化湖泊 (如巢湖 ${ }^{[23]}$ 、太湖 ${ }^{[24]}$ 、东湖 ${ }^{[11]}$ 等) 相 比, 种类数明显低于后者. 此外, 按照相关资料 ${ }^{[7,21]}$, 计算得出 18 个物种的平均耐污值高达 7.14 , 其中苏氏尾 鳃蚛、霍甫水丝蚓、克拉伯水丝蚓和长足摇蚊等均具有很强的耐污性, 均是富营养化水体的常见优势种 ${ }^{[25]}$. 密度和生物量方面, 珠江三角洲池塘平均分别为 $14331 \mathrm{ind} . / \mathrm{m}^{2}$ 和 $35.53 \mathrm{~g} / \mathrm{m}^{2}$. 由于缺乏软体动物, 因此生物 
量比一些河流或通江湖泊明显要低 ${ }^{[26-27]}$, 但密度却远高于长江中下游一些严重富营养化湖泊 ${ }^{[11,24]}$. 就 6 种 养殖模式而言, 虽然其种类组成差异不大, 但优势种以及不同类群密度和生物量所占的比例却有较大差异. 在草鱼和罗非鱼池塘, 优势种仅为塞毛类; 而在其他 4 种类型池塘中, 优势种主要为塞毛类和摇蚊幼虫. 所 占比例方面, 除了罗非鱼池塘软体动物生物量所占比例较大外, 其他池塘密度和生物量均以寡毛类和水生 昆虫 (主要是摇蚊幼虫) 为主. 塞毛类和摇蚊幼虫均为水体富营养化的常见指示生物 ${ }^{[28]}$, 一些研究已经表明 二者均喜欢有机质丰富的淤泥底质, 富营养化水体中有机质浓度的增加会明显导致摇蚊幼虫与塞毛类 (特 别是颤蚓科) 密度和生物量的提高 ${ }^{[25,29]}$. 罗非鱼池塘是 6 种模式中养殖密度最低的 (仅为杂交鳢养殖密度的 $1 / 17$ ), 其投饵量较低, 受污染程度也较低, 因此软体动物生物量相对较高. 4 种软体动物中的 2 种一一长角 涵螺和放逸短沟蜷均出现在此类型池塘.

方差分析结果表明, 6 种养殖模式池塘底栖动物密度和生物量均存在显著差异, 且杂交鳢 $\mathrm{Y}$ 和杂交鳢 $\mathrm{C}$ 养殖池塘显著高于其他 4 种养殖模式池塘. 该结果间接表明 2 种杂交鳢养殖模式池塘可能受到的污染最为 严重, 而这种现象可能主要是由两方面造成的, 一是杂交鳢池塘养殖密度大、投饵量大, 二是杂交鳢池塘所 用饲料的粗蛋白和总磷浓度要明显高于草鱼和罗非鱼池塘 (表 1). 此外, 随着养殖密度的增加, 4 种鱼类池 塘底栖动物密度和生物量还表现出逐渐增加的趋势; 这表明池塘底栖动物可能受鱼类养殖品种的影响较 小, 而受投饵量以及饲料中营养物质浓度的影响较大, 因为这几种鱼类都很少会直接以底泥中的寡毛类或 摇蚁幼虫为食. 2 种大口黑鲇 (大口黑鲇 $\mathrm{S}$ 和大口黑鲇 $\mathrm{S}+\mathrm{B}$ ) 池塘底栖动物密度和生物量无显著差异, 表明 投喂冰鲜鱼类并不会明显改变底栖动物的现存量; 而 2 种杂交鳢 (杂交鳢 $\mathrm{Y}$ 和杂交鳢 $\mathrm{C}$ ) 池塘也无显著差 异,则说明鱼类的养殖规格对底栖动物的影响也较小.

\section{2 影响因素}

影响底栖动物群落结构的因素众多, 但在富营养化的静态水体中, 底质类型、底泥或水体中 TN 和 TP 浓 度等是常见的主要影响因素. 底质是底栖动物生存的基本条件, 可为其提供多样的栖息地环境, 对其生长、 繁殖等重要生命阶段都起着关键作用,这主要与底质的粒径、异质性、稳定程度、密实性、颗粒间隙和表面结 构等有关 ${ }^{[30]}$. 如, 对富营养化湖泊巢湖 ${ }^{[23]}$ 、太湖 ${ }^{[24]}$ 的研究均表明, 底栖动物的现存量与底质类型呈显著相 关. 然而, 本研究中所调查的 30 口池塘底质异质性较低, 且都主要是以淤泥为主, 所以底质可能不是池塘中 底栖动物的主要影响因素. 关于 TN 和 TP 浓度与底栖动物的关系, 许多研究均已表明水体的富营养化程度 越高, 营养盐对底栖动物的影响就越显著 ${ }^{[25]}$, 且底栖动物现存量一般与 TN 、 TP 浓度呈显著正相关 ${ }^{[25,31]}$. 本 研究中, 对珠江三角洲池塘的研究结果与此一致. 就不同分类群而言, 由于寡毛类和摇蚊幼虫等直接收集者 均喜欢有机质丰富、淤泥底质的环境 ${ }^{[23]}$, 臭毛类 (主要是颤蚛类) 和摇蚊幼虫的现存量一般也与水体的 TN 和 TP 浓度呈显著正相关 ${ }^{[25]}$. 姜苹红等 ${ }^{[25]}$ 还以江汉湖群为例, 建立了以上 2 种类群与水体营养盐的回归模 型. 而本研究中, 摇蚊幼虫的研究结果与此一致, 而寡毛类的密度和生物量却仅与 TN 浓度呈显著正相关, 这 可能主要是由于池塘这种小水体理化参数相对不稳定, 在较短时间内可能有较大的变化. 比如一些水质改 良剂、杀菌药物在短时间内均能明显改变水体中营养盐浓度, 这也间接表明在小水体中生物监测可能比化 学监测更能反映出水体的健康状况.

\section{3 水质评价}

目前用于水质生物评价的指数较多, 由于不同指数划分水质的等级不同, 因而得出的评价结果常存在 一定的差异. 本文首次采用常用的 3 个指数, 即 Shannon-Wiener 多样性指数、Margalef 多样性指数和 $B I$ 生物 指数对珠江三角洲池塘水质作出了评价, 评价结果也存在一定的差异. Shannon-Wiener 多样性指数评价结果 与实际情况差距较大, 这主要是由于该指数一般在水质差异较大的水体中评价效果较好, 而不能分辨水质 差异较小的水体. 此外, 该指数还认为各物种在群落中的地位平等, 从而忽视了不同物种对污染的耐受能力 和敏感性差异, 因此很多情况下不能真实反映水质的实际情况 ${ }^{[19]}$. 国内对东湖 ${ }^{[11]}$ 、丹江口水库 ${ }^{[13]}$ 和矿区塌 陷湖 ${ }^{[6]}$ 的研究也有类似结果, 因此不推荐使用该指数用于珠江三角洲富营养化池塘的水质评价. Margalef 多 样性指数和 $B I$ 生物指数评价结果认为少部分池塘属于中度污染, 大部分池塘属于重度污染, 评价结果要优 于 Shannon-Wiener 多样性指数. 以 6 种类型池塘评价指数的平均值作为比较参数, Margalef 多样性指数评价 的水质优劣顺序为: 罗非鱼>鲇鱼 $\mathrm{S}>$ 鲇鱼 $\mathrm{S}+\mathrm{B}>$ 杂交鳢 $\mathrm{Y}>$ 杂交鳢 $\mathrm{C}>$ 草鱼, 而 $B I$ 指数评价的优劣顺序为: 罗 
非鱼>鲇鱼 $\mathrm{S}+\mathrm{B}>$ 鲇鱼 $\mathrm{S}>$ 草鱼>杂交鳢 $\mathrm{Y}>$ 杂交鳢 $\mathrm{C}$. 二者相比较而言, $B I$ 指数的评价结果可能要优于 Margalef 多样性指数, 并且与已有的池塘水化学评价结果一致 ${ }^{[2]}$. 因此, 我们推荐 $B I$ 指数作为珠江三角洲池 塘水质评价的首选指数.

致谢: 中国水产科学研究院珠江水产研究所李家磊、付兵等在野外采样和水化学测定中给予帮助,特此 感谢!

\section{4 参考文献}

[ 1 ] Zhong Gongpu, Deng Hanzeng, Wu Houshui et al eds. Dike-pond system in the Pearl River Delta. Beijing: Science Press, 1987: 98-115. [钟功甫, 邓汉增, 吴厚水等. 珠江三角洲基塘系统研究. 北京:科学出版社, 1987:98-115.]

[ 2 ] Liu Qianfu, Lai Zini, Yang Wanling et al. Assessment of water quality of intensive ponds in the Pearl River Delta region. South China Fisheries Science, 2014, 10(6) : 36-43. [刘乾甫, 赖子尼, 杨婉玲等. 珠三角地区密养淡水鱼塘水质状 况分析与评价. 南方水产科学, $2014,10(6): 36-43$.

[ 3 ] Liu Feng, Li Xiuqi, Wang Fang et al. Review on budgets and environmental loads of nitrogen and phosphorus in culture system. Marine Environmental Science, 2011, 30(4) : 603-608. [刘峰, 李秀启, 王芳等. 养殖系统 N、P 收支及环境 N、P 负荷量的研究进展. 海洋环境科学, 2011, 30 (4) : 603-608.]

[ 4 ] Wetzel RG ed. Limnology: Lake and river ecosystems (3rd ed). San Diego: Academic Press, 2001.

[ 5 ] Barbour MT, Gerritsen J, Snyder BD et al eds. Rapid bioassessment protocols for use in streams and wadeable rivers: Periphyton, benthic macroinvertebrates, and fish (2nd ed). Washington, DC: USEPA, 1999.

[ 6 ] Ji Lei, Li Xiaoming, Deng Daogui. Community structure of macrozoobenthos and bioassessment of water quality in collapse lakes in huaibei mining areas. Acta Hydrobiologica Sinica, 2016, 40(1): 147-156. [纪否, 李晓明, 邓道贵. 淮北煤矿 区塌陷湖大型底栖动物群落结构及水质生物学评价. 水生生物学报, 2016, 40(1): 147-156.]

[ 7 ] Wang Beixin. Water quality bioassessment using benthic macroinvertebrates [Dissertation]. Nanjing: Nanjing Agricultural University, 2003. [王备新. 大型底栖无脊椎动物水质生物评价研究 [学位论文]. 南京: 南京农业大学, 2003.]

[ 8 ] Xia Aijun, Chen Xiaohui, Cai Yongxiang et al. The status of zoobenthos community structure and preliminary evaluation of water quality in the Jiangsu section of the Yangtze River. Marine Fisheries, 2006, 28(4): 272-277. [夏爱军, 陈校辉, 蔡永祥等. 长江江苏段底栖动物群落结构现状及其水质的初步评价. 海洋渔业, 2006, 28(4) : 272-277.]

[ 9 ] Liu XQ, Gippel CJ, Wang HZ et al. Assessment of the ecological health of heavily utilized, large lowland rivers: Example of the lower Yellow River, China. Limnology, 2016,18(1):1-13.

[10] Liu Lusan, Li Zhongyu, Meng Wei et al. The community structure of zoobenthos and bioassessment of water quality in the lower reaches of the Songhua River. Research of Environmental Sciences, 2007, 20(3):81-86. [刘录三, 李中宇, 孟伟 等. 松花江下游底栖动物群落结构与水质生物学评价. 环境科学研究, 2007, 20(3): 81-86.]

[11] Wang Qin, Wang Haijun, Cui Yongde et al. Community characteristics of the macrozoobenthos and bioassessment of water quality in lake Donghu district, Wuhan. Acta Hydrobiologica Sinica, 2010, 34(4): 739-746. [王琴, 王海军, 崔永德. 武汉东湖水网区底栖动物群落特征及其水质的生物学评价. 水生生物学报, 2010, 34(4): 739-746.]

[12] Dai Youzhi, Tan Shouyin, Zhang Jianbo. The distribution of zoobenthos species and bio-assessment of water quality in Dongting Lake. Acta Ecologica Sinica, 2000, 20(2) : 277-282. [戴友芝, 唐受印, 张建波. 洞庭湖底栖动物种类分布 及水质生物学评价. 生态学报, $2000,20(2): 277-282$. ]

[13] Zhang Min, Shao Meiling, Cai Qinghua et al. Macroinvertebrate community structure and the biological assessment to the water quality of the Danjiangkou Reservoir. J Lake Sci, 2010, 22(2) : 281-290. DOI : 10.18307/2010.0219. [ 张敏, 邵美 玲, 蔡庆华等. 丹江口水库大型底栖动物群落结构及其水质生物学评价. 湖泊科学, 2010, 22(2): 281-290.]

[14] Liu Yueying, Zhang Wenzhen, Wang Yaoxian eds. Freshwater Mollusk Economic Fauna of China. Beijing: Science Press, 1979: 6-126. [刘月英, 张文珍, 王耀先. 中国经济动物志(淡水软体动物). 北京:科学出版社, 1979:6-126.]

[15] Morse JC, Yang LF, Tian LX eds. Aquatic insects of China useful for monitoring water quality. Nanjing: Hohai University Press, 1994:10-562.

[16] Wang Hongzhu eds. Studies on taxonomy, distribution and ecology of microdrile Oligochaetes of China, with descriptions of two new species from the vicinity of the Great Wall station of China, Antarctica. Beijing: Higher Education Press, 2002:1225. [王洪铸. 中国小蚓类研究——附中国南极长城站附近地区两新种. 北京: 高等教育出版社, 2002:1-225.] 
[17] Pan B, Wang H, Wang H et al. Macrozoobenthic assemblages in relation to environments of the Yangtze-isolated lakes. Frontiers of Environmental Science and Engineering, 2012, 6(2) : 246-254.

[18] Ministry of Environmental Protection of the People's Republic of China, Editorial Board of Water and Wastewater Monitoring and Analysis Methods ed. Analysis in water and wastewater. Beijing: China Environmental Science Press, 2002. [国 家环境保护总局《水和废水监测分析方法》编委会. 水和废水监测分析方法. 北京: 中国环境科学出版社, 2002.]

[19] Shannon CE, Wiener WJ eds. The mathematical theory of communication. Urbana: University of Illinois Press, 1949 : 296.

[20] Margalef DR. Information theory in ecology. Generation Systems, 1957, 3: 36-71.

[21] Qin CY, Zhou J, Cao Y et al. Quantitative tolerance values for common stream benthic macroinvertebrates in the Yangtze River Delta, Eastern China. Environmental Monitoring and Assessment, 2014, 186(9): 1-13.

[22] Jackson C, Preston N, Thompson P et al. Nitrogen budget and effluent Nitrogen components at an intensive Shrimp farm. Aquaculture, 2003 , 218(1-4) : 397-411.

[23] Zhao Yongjing, Liu Xueqin. Community Structure of macrozoobenthos in the littoral zone of the Chaohu Lake. Resources and Environment in the Yangtze Basin, 2012, 21(Z2): 39-44. [ 赵永晶, 刘学勤. 巢湖水向湖滨带底栖动物群落结构 研究. 长江流域资源与环境, 2012, 21(Z2): 39-44.]

[24] Cai Yongjiu, Gong Zhijun, Qin Boqiang. Community structure and diversity of macrozoobenthos in Lake Taihu, a large shallow eutrophic lake in China. Biodiversity Science, 2010, 18(1):50-59. [蔡永久, 龚志军, 秦伯强. 太湖大型底栖 动物群落结构及多样性. 生物多样性, 2010, 18(1) : 50-59.]

[25] Jiang Pinghong, Cui Yongde, Wang Haijun et al. Macrozoobenthos communities and bio-assessment of lakes in Hanyang district. Resources and Environment in the Yangtze Basin, 2011, 20(5): 525-533. [姜苹红, 崔永德, 王海军等. 汉阳湖 群底栖动物群落及其对环境质量的指示. 长江流域资源与环境, 2011，20(5)：525-533.]

[26] Xie Zhicai, Zhang Junqian, Chen Jing et al. Spacial distributional pattern of macrozoobenthos and pollution evaluation in East Lake Dongting reserve. J Lake Sci, 2007, 19(3) : 289-298. DOI:10.18307/2007.0310. [谢志才, 张君倩, 陈静等. 东洞庭湖保护区大型底栖动物空间分布格局及水质评价. 湖泊科学, 2007, 19(3): 289-298.]

[27] Zhang Xiaoke, Xuan Hao, Wang Huili et al. Macrozoobenthos community structure in different order streams of the Qingyi river basin. Resources and Environment in the Yangtze Basin, 2014, 23(12) : 1659-1664. [张晓可, 宣吴, 王慧丽等. 青 七江流域不同级别河流底栖动物群落结构研究. 长江流域资源与环境, 2014, 23(12): 1659-1664.]

[28] Gong Zhijun, Xie Ping, Tang Huijuan et al. The influence of eutrophycation upon community structure and biodiversity of Macrozoobenthos. Acta Hydrobiologica Sinica, 2001, 25(3): 210-216. [龚志军, 谢平, 唐汇涓等. 水体富营养化对大 型底栖动物群落结构及多样性的影响. 水生生物学报, 2001, 25(3): 210-216.]

[29] Peralta L, Escobar E, Alcocer J et al. Oligochaetes from six tropical crater lakes in Central Mexico: Species composition, density and biomass. Hydrobiologia, 2002, 467(1/2/3) : 109-116.

[30] Duan Xuehua, Wang Zhaoyin, Xue Mengzhen eds. Benthic macroinvertebrate and application in the assessment of stream ecology. Bingjing: Tsinghua University Press, 2010. [段学花, 王兆印, 徐梦珍. 底栖动物与河流生态评价. 北京: 清 华大学出版社, 2010.]

[31] Jiang Pinghong, Liang Xiaomin, Chen Fang et al. Indication of macrophyte- restorable area by spatial pattern of macrobenthos in Yuehu Lake. Resources and Environment in the Yangtze Basin, 2006, 15(4): 502-505. [姜苹红, 梁小民, 陈 芳等. 月湖底栖动物的空间格局及其对水草可恢复区的指示. 长江流域资源与环境, 2006, 15(4): 502-505. ] 